\title{
Optical Absorption of hexagonal Boron Nitride and $B N$ nanotubes
}

\author{
Ludger Wirtz*, Andrea Marini ${ }^{\dagger}$ and Angel Rubio** \\ *Institute for Electronics, Microelectronics, and Nanotechnology (IEMN CNRS-UMR 8520), \\ B.P. 60069, 59652 Villeneuve d'Ascq Cedex, France \\ ${ }^{\dagger}$ Istituto Nazionale per la Fisica della Materia e Dipartimento di Fisica dell'Universitá di Roma \\ "Tor Vergata", Via della Ricerca Scientifica, I-00133 Roma, Italy \\ ${ }^{*}$ Department of Material Physics, University of the Basque Country, Centro Mixto CSIC-UPV, \\ and Donostia International Physics Center (DIPC), Po. Manuel de Lardizabal 4, 20018
}

Donostia-San Sebastián, Spain

\begin{abstract}
We present calculations for the optical absorption spectra of hexagonal boron nitride $(\mathrm{hBN})$ and $\mathrm{BN}$ nanotubes, using many-body perturbation theory. Solution of the Bethe-Salpeter equation for $\mathrm{hBN}$ leads to optical absorption and loss spectra where the positions and shapes of the peaks are strongly dominated by excitonic effects. The binding energy of the first exciton is about $0.71 \mathrm{eV}$. Comparison of the calculations with recently measured optical absorption and EELS demonstrates that DFT underestimates the "true" band gap of BN by $2.25 \mathrm{eV}$. This band gap difference can be partially (but not completely) reproduced by a calculation of the quasi-particle band-structure on the level of the GW-approximation. We show how the lower dimensionality of $\mathrm{BN}$ nanotubes leads to a much stronger excitonic binding energy and at the same time to a larger quasi-particle gap. This leaves the position of the first absorption peak almost unchanged. However, the difference in the series of excitonic peaks allows the spectroscopic distinction between BN nanotubes and bulk BN.
\end{abstract}

Keywords: BN nanotubes, excitonic effects, Bethe-Salpeter equation, GW-approximation PACS: 78.67.Ch, 78.20.Bh, 71.35.Cc, 71.45.Gm

\section{INTRODUCTION}

Hexagonal boron nitride $(\mathrm{hBN})$ is isoelectronic to graphite and has the same layered structure (only with different stacking). Graphite is a semi-metal with a linear crossing of the $\pi$ and $\pi^{*}$ bands at the K-point of the Brillouin zone. In $\mathrm{hBN}$, however, the different electronegativities of boron and nitrogen lift the degeneracy at the K-point and lead to a considerable gap of $4.5 \mathrm{eV}$ (in the LDA). The optical properties of hBN have been investigated experimentally (e.g. [1, 2]) and theoretically (e.g., [3, 4]) on the level of the Random Phase Approximation (RPA). Recently, hBN has regained interest due to the discovery of BN-nanotubes [5, 6] which can be considered as cylinders formed by rolling a single sheet of $\mathrm{hBN}$ onto itself. In the RPA, optical absorption is modeled as independent electron transitions from the valence to the conduction band. Since the electronic structure of the tubes can be constructed by rolling the sheet, the RPA optical absorption spectra for the 3-dimensional bulk-BN, for 2-dimensional single sheet and for the (quasi-)one dimensional tubes are very similar ([7, 8, 9] and see Fig. 2), for light polarization parallel to the layer-plane and tube axis and strong broadening of the spectra. For large radius tubes, the absorption spectrum must be the same as for the 
sheet, since the electronic structure converges towards the one of the sheet, and even for tubes with small radius, the presence of 1-dimensional van-Hove singularities in the density of states gives only small additional fine-structure in a spectrum that essentially resembles the one of the plane. Going beyond the simplistic picture of independent particle transitions and using the techniques of many-body perturbation theory, we will show that the optical properties of $\mathrm{hBN}$ are strongly dominated by excitonic effects. The difference in excitonic binding energies between $\mathrm{hBN}$ and the tubes will thereby lead to measurable differences in the optical spectra. The influence of excitonic effects in carbon nanotubes was recently shown $[10,11]$.

\section{METHODS}

Our calculations of optical absorption spectra, which are proportional to the imaginary part of the dielectric function, $\varepsilon_{2}(E)$, proceed in three steps:

I.) First we perform a self-consistent calculation of the (geometry-optimized) ground state density using density functional theory (DFT) in the local-density approximation (LDA). Then we calculate the Kohn-Sham wavefunctions $\psi_{n k}$ of the occupied and a large number of unoccupied states (with a band-index $n$ and a sufficiently fine discrete $k$-point sampling of the first Brillouin zone in reciprocal space). The calculations are performed with the code ABINIT [12]. Core electrons are substituted by pseudo-potentials and valence electrons are expanded in plane waves. Frequently, the LDA wavefunctions and energies are used directly to calculate the matrix elements for optical transitions. This corresponds to the random-phase approximation (RPA). For many materials, however, the thus-obtained spectra are in qualitative and quantitative disagreement with measured absorption spectra [13] and further calculations using the techniques of many-body perturbation theory are necessary. This is also the case for $\mathrm{hBN}$ and $\mathrm{BN}$ nanotubes.

II.) Within the GW-approximation [13], we calculate the quasi-particle energies ("true" single-particle excitation energies), $E_{n k}$, by solving the Dyson equation

$$
\left[-\frac{\nabla^{2}}{2}+V_{\text {ext }}+V_{\text {Hartree }}+\Sigma\left(E_{n k}\right)\right] \psi_{n k}=E_{n k} \psi_{n k}
$$

The self-energy $\Sigma=i G W$ is approximated as the product of the one-particle Green's function $G$ and the screened Coulomb interaction $W$. The $\mathrm{GW}$-approximation increases considerably the LDA-band gap.

III.) An electron that is excited from the valence band to the conduction band can interact with the remaining "hole" through a screened Coulomb potential. This can lead to bound, excitonic, states within the band-gap. Quantitatively, the excitonic levels are calculated by the Bethe-Salpeter equation

$$
\left(E_{c k}-E_{v k}\right) A_{v c k}^{S}+\Sigma_{k^{\prime} v^{\prime} c^{\prime}}\left\langle v c k\left|K_{e h}\right| v^{\prime} c^{\prime} k^{\prime}\right\rangle A_{v^{\prime} c^{\prime} k^{\prime}}^{S}=\Omega^{S} A_{v c k}^{S}
$$

The electron-hole interaction kernel $K_{e h}$ "mixes" different electron transitions from valence band states $v, v^{\prime}$ to conduction band states $c, c^{\prime}$. This leads to transition energies $\Omega^{s}$ that can be quite different from the quasiparticle-energy differences $E_{c k}-E_{v k}$. The presence of bound excitons can lead to a considerable red-shift of oscillator strength. The calculations of step II and III are performed with the code SEIF [16]. 


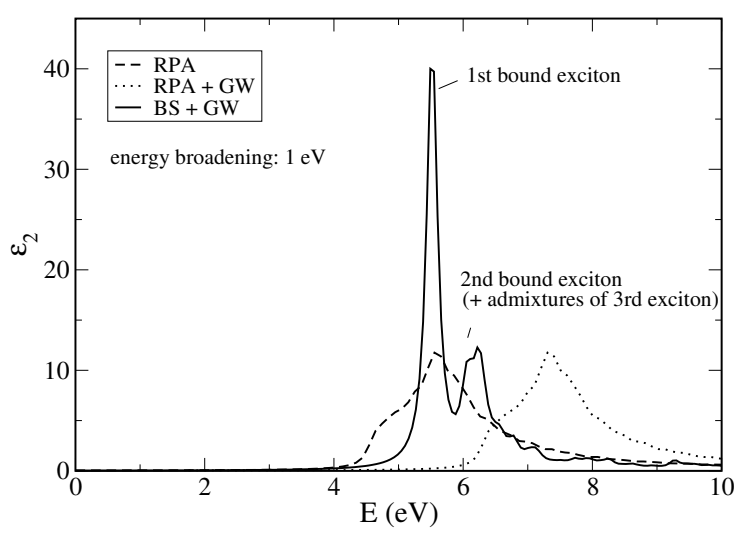

FIGURE 1. Optical absorption of bulk hBN calculated on three levels of approximation (see text).

\section{RESULTS}

In Fig. 1, we compare the results of the three levels of calculation for hBN [17]. The light-polarization is chosen parallel to the plane. The spectra are calculated with a Lorentzian broadening of $0.1 \mathrm{eV}$, corresponding approximately to experimental values for broadening. The dashed line shows the result of the RPA calculation (compare [3] and [4]). The broad peak with a maximum at $5.6 \mathrm{eV}$ is entirely due to the continuum of inter-band transitions between the $\pi$ and $\pi^{*}$ bands. (Transitions between $\pi$ and $\sigma$ bands are forbidden due to selection rules). The GW-calculation leads to an almost uniform downward shift of the $\pi$ band and upward shift of the $\pi^{*}$ band (compare [14]). This results in a blue-shift with respect to the RPA-absorption spectrum by $1.7 \mathrm{eV}$ while the shape remains approximately the same (dotted line). An important change takes place when we include excitonic effects through the Bethe-Salpeter (BS) equation. This leads to a the double-peak spectrum (solid line in Fig. 1) with the high peak at almost the same position as the maximum in the RPA-spectrum. The blue-shift due to the GW corrections to the band-structure and the red-shift due to excitonic effects thus almost cancel each other. However, the shape of the spectrum has changed considerably. Contrary to the RPA spectrum which is due to a continuum of inter-band transitions, the BS spectrum is dominated by two discrete bound excitons. This can be seen more clearly in Fig. 2 A), where the same spectrum, but with a broadening of $0.025 \mathrm{eV}$, is plotted. There is a sequence of bound excitonic peaks below the onset of the continuum (which is determined by the GW-band gap). Most of the oscillator strength is collected by the first peak while at the onset of the continuum almost no absorption takes place. The binding energy of the first exciton is $0.71 \mathrm{eV}$. We note that the shape of the broadened BS spectrum of Fig. 1 is in very good agreement with the experimental data of Tarrio and Schnatterly (Ref. [1], Fig. 2, which was obtained by a Kramers-Kroning transform of the electron-energy loss function). It also matches well the optical absorption spectrum of $\mathrm{hBN}$ of Ref. [2]. Only the position of the BS+GW spectrum comes out too low by 0.55 $\mathrm{eV}$ in comparison with experimental data. We suppose that this is due to the limited accuracy of the GW-approximation and that the quasiparticle correction to the LDA band-gap should be rather $2.25 \mathrm{eV}$ than the calculated shift of $1.7 \mathrm{eV}$ mentioned above. 

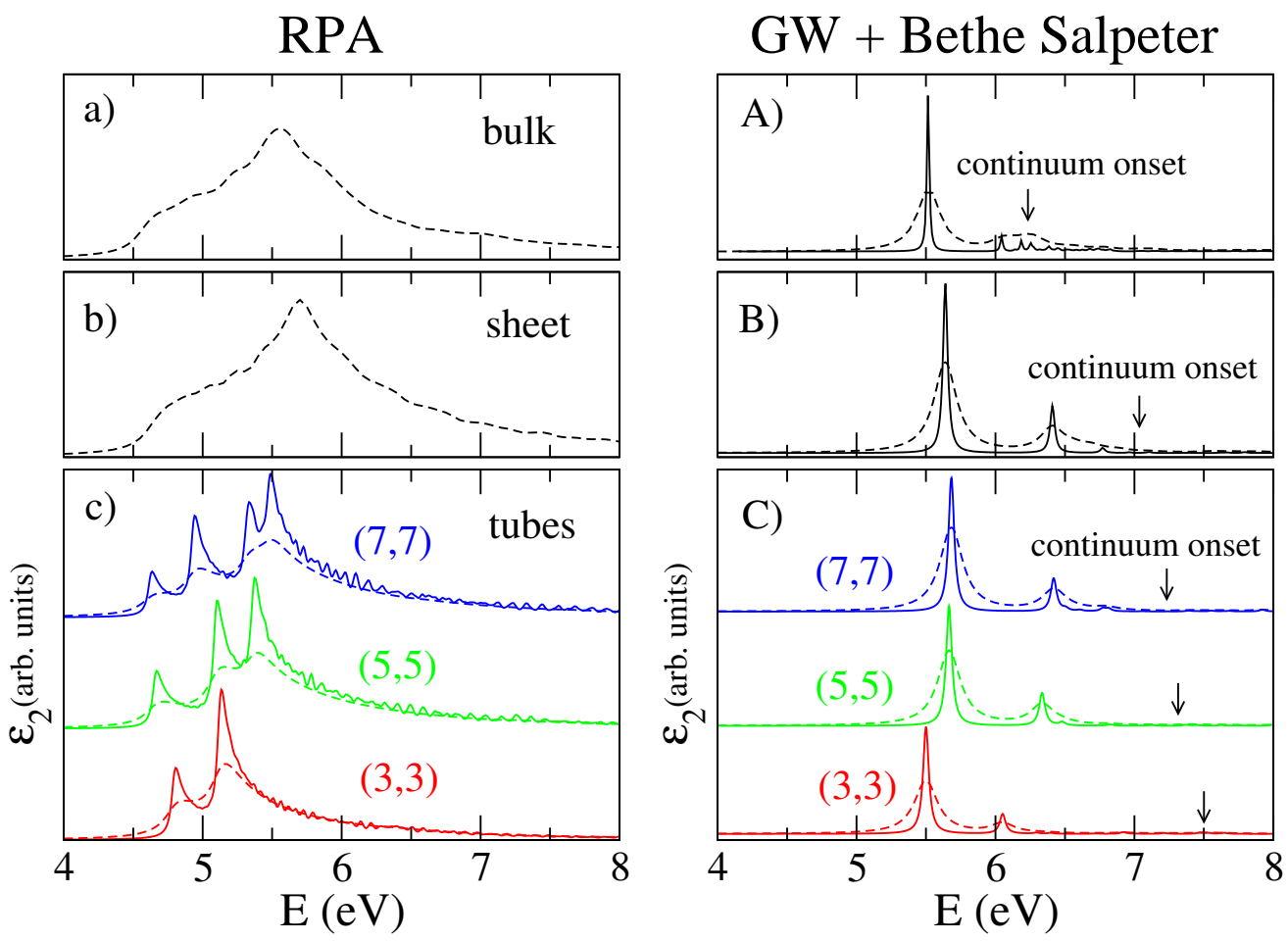

FIGURE 2. Optical absorption of hBN (a), BN-sheet (b) and three different BN tubes (c). We compare the results of the $\mathrm{GW}+$ Bethe-Salpeter approach (right hand side) with the random phase approximation (left hand side). Solid lines are calculated with a Lorentzian broadening of $0.025 \mathrm{eV}$, dashed lines with a broadening of $0.1 \mathrm{eV}$ (for comparison with experimental data). The light polarization is parallel to the plane/ tube axis, respectively.

Keeping this limitation in mind, we conclude that the GW+BS method is well suited to explain the shape of the optical absorption (and EELS) spectra of hBN. We apply it consequently for the calculation of the spectra of the (quasi) two-dimensional BN sheet and the (quasi) 1-dimensional BN nanotubes. Our theoretical data is also in very good agreement with a recent EELS measurement of multiwalled BN-nanotubes [15].

On the left-hand side of Fig. 2 we present the RPA spectra of bulk hBN, of the singlesheet of hBN (calculated in a periodic supercell with an inter-sheet distance 20 a.u.), and of three different BN nanotubes (supercell with inter-tube distance of 20 a.u.) [18]. The RPA spectra of the bulk and the single sheet are almost indistinguishable. For the tubes, the RPA spectra display some peak structure below $5.5 \mathrm{eV}$ which is due to the van-Hove singularities in the one-dimensional density of states. For tubes with larger radii, the density of the fine-structure peaks increases and the RPA spectrum approaches that of the sheet. Note that the fine structure is only visible in the calculation with small broadening (solid lines). In the calculation with $0.1 \mathrm{eV}$ broadening, the RPA spectrum of the tubes is almost indistinguishable from the sheet and the bulk (compare [8] and [9]). As was already discussed for bulk $\mathrm{BN}$, the BS+GW spectra are entirely different in shape, because they are dominated by excitonic peaks. The reduced dimensionality leads to a stronger binding energy of the first exciton. In the two-dimensional sheet we obtain a binding energy of $1.4 \mathrm{eV}$ as compared with the bulk value of $0.71 \mathrm{eV}$. For the 
small diameter, quasi-one dimensional, $\mathrm{BN}(3,3)$ tube the binding energy even reaches 2 $\mathrm{eV}$. With increasing tube diameter, the binding energy converges to the binding energy of the sheet $(1.62 \mathrm{eV}$ for the $(5,5)$ tube and $1.58 \mathrm{eV}$ for the $(7,7)$ tube. At the same time, the reduced dimensionality leads to an increase of the GW gap [19]. Therefore, the position of the dominant absorption peak (the first excitonic peak) remains almost unchanged. The distance between the first and second excitonic peak increases with increasing tube diameter and rapidly converges towards the distance of the excitonic peaks in the sheet. This is an indication that the exciton is confined in a small region (a few atomic distances) and feels only the "local environment" Only in the strongly curved $(3,3)$ tube, the exciton can be delocalized over the whole circumference of the tube. In the larger tube, the exciton only "sees" the locally flat environment as in a sheet.

In conclusion, the optical absorption spectra of $\mathrm{hBN}$ and $\mathrm{BN}$ nanotubes are dominated by excitonic effects. Most oscillator strength is collected by the first bound exciton. The binding energy of this peak increases strongly as the dimensionality is reduced from the $3-\mathrm{D}$ bulk over the 2-D sheet to the 1-D tubes. At the same time the quasi-particle band gap increases with reduced dimensionality. This cancellation leaves the absolute position of the dominant absorption peak almost constant. Differences can be seen, however, in the energy differences between the excitonic peaks. We acknowledge support by the EU Network of Excellence NANOQUANTA (NMP4-CT-2004-500198). Calculations were performed at IDRIS (Project No. 51827) and CEPBA.

\section{REFERENCES}

1. C. Tarrio and S. E. Schnatterly, Phys. Rev. B 40, 7852 (1989).

2. J. S. Lauret et al., Phys. Rev. Lett. 94, 037405 (2005).

3. Y. Xu and W. Y. Ching, Phys. Rev. B 44, 7787 (1991).

4. G. Cappellini, G. Satta, M. Palummo, and G. Onida, Phys. Rev. B 64, 035104 (2001).

5. A. Rubio, J. L. Corkill, and M. L. Cohen, Phys. Rev. B 49, R5081 (1994); X. Blase, A. Rubio, S. G. Louie, and M. L. Cohen, Europhys. Lett 28, 335 (1994).

6. N. G. Chopra et al., Science 269, 966 (1995).

7. L. Wirtz, V. Olevano, A. G. Marinopoulos, L. Reining, and A. Rubio, AIP Conf. Proc. 685, 406 (2003).

8. A. G. Marinopoulos et al., and L. Reining, Appl. Phys. A 78, 1157 (2004).

9. G.Y. Guo and J.C. Lin, Phys. Rev. B 71, 165402 (2005).

10. C. D. Spataru et al., Phys. Rev. Lett. 92, 077402 (2004); Appl. Phys. A 78, 1129 (2004).

11. E. Chang, G. Bussi, A. Ruini, and E. Molinari, Phys. Rev. Lett. 92, 196401 (2004).

12. X. Gonze et al., Comp. Mat. Sci. 25, 478 (2002).

13. See, e.g., G. Onida, L. Reining, and A. Rubio, Rev. Mod. Phys. 74, 601 (2002).

14. X. Blase, A. Rubio, S.G. Louie and M. L. Cohen, Phys. Rev. B 51, 6868 (1995)

15. G. G. Fuentes et al., Phys. Rev. B 67, 035429 (2003).

16. SETF , written by A. Marini (http://people.roma2.infn.it/ marini/self/)

17. Energy minimization leads to a bond-length of $1.441 \AA$ and an inter-sheet distance of $3.251 \AA$. Energies and wave-functions are evaluated with an energy cutoff at 25 Hartree, using a 40x40x12 Monkhorst-Pack grid in reciprocal space. In the GW and BS calculations, we used a 16x16x6 Monkhorst-Pack sampling which is sufficient to converge the dominant discrete excitonic peaks. Unoccupied states with energies up to $40 \mathrm{eV}$ above the band-gap are included.

18. The tubes are geometry-optimized. For the GW+BS calculations, the 1-D Brillouin zone is sampled by $20 \mathrm{k}$-points (corresponding to 11 irreducible k-points). Convergence of the spectra with respect to this number was checked numerically.

19. C. Delerue, G. Allan, and M. Lannoo, Phys. Rev. Lett. 90, 076803 (2003) 